\title{
ON A CONJECTURE OF D. C. MURDOCH CONCERNING PRIMARY DECOMPOSITIONS OF AN IDEAL
}

\author{
KIM LIN CHEW ${ }^{1}$
}

Regarding the existence of a right primary decomposition of an ideal $A$ in a ring $R$ with ascending chain condition on ideals, Murdoch has suggested the conjecture that if every prime ideal $P$ which is not right prime to the right upper $P$-component $u(A, P)$ occurs as an associated prime of $A$, then $A$ has a right primary decomposition $[7$, p. 739]. A counterexample to this conjecture is furnished in the first part of this paper while the latter part gives a number of criteria for a ring to have a Noetherian ideal theory and extends the recent Barnes-Cunnea construction [2] of the primary decomposition to a noncommutative ring.

1. Notations and terminology. Definitions are precisely those given in $[7, \S 8]$, except that, for the sake of easy computation, we formulate them in terms of ideals rather than of elements. Under set theoretical inclusion, the family $L(R)$ of all ideals of a ring $R$ becomes a complete modular lattice with an associative, join distributive multiplication, a left residuation $(:)$ and a right residuation $(::)$ defined as follows:

$$
A: B=\sum\{X \in L(R) \mid X B \subseteq A\}, \quad A:: B=\sum\{X \in L(R) \mid B X \subseteq A\} .
$$

An ideal $P$ of $R$ is said to be prime if $P: X=P:: X=P$ for any $X \nsubseteq P$. The intersection of all prime ideals containing $A \in L(R)$ is called the radical of $A$ and is denoted by $\operatorname{rad} A$. In case $\operatorname{rad} A$ is a prime ideal $P, A$ is said to be quasi $P$-primary. An ideal $Q$ of $R$ is said to be (right) primary if $Q:: X=Q$ for any $X \nsubseteq \operatorname{rad} Q$. By [6, Theorem $10]$, if $L(R)$ satisfies the ascending chain condition, then every ideal contains a power of its radical. Accordingly the radical of a primary ideal is prime.

An ideal $T$ of $R$ is said to be (right) tertiary if $T:: X=T$ for any $X \nsubseteq t$-rad $T$, where $t$-rad $T$ denotes the ideal $\sum\{X \in L(R) \mid(T:: X) \cap Y$ $=T$ implies $Y=T\}$ and is called the (right) tertiary radical of $T$. In case $L(R)$ satisfies the ascending chain condition, Lesieur and Croisot [4] have succeeded in showing that the tertiary radical of a tertiary ideal is prime, that every ideal $A$ can be represented as an irredun-

Received by the editors March 21, 1967.

1 The author is indebted to Professor D. C. Murdoch for his encouragement and to the referee for his valuable suggestions. 
dant intersection of a finite number of tertiary ideals whose tertiary radicals (called the associated primes of $A$ ) are distinct, and that $t$-rad $A$ is the intersection of the associated primes of $A$. Consequently $t$-rad $A \supseteq \operatorname{rad} A$ and every primary ideal is tertiary.

An element $x$ of $R$ is (right) prime to $A$ if $A::(x)=A$, where $(x)$ denotes the principal ideal in $R$ generated by $x$. It is said to be not (right) prime to $A$ otherwise. An ideal $B$ is said to be not (right) prime to $A$ if every element of $B$ is not prime to $A$. If $A:: B \neq A$, then evidently $B$ is not prime to $A$. The converse is true if $R$ satisfies the ascending chain condition on ideals [1, Theorem 10]. For a prime ideal $P$ of $R$ the (right) upper $P$-component $u(A, P)$ of $A$ is defined to be the intersection of all ideals $B$ containing $A$ such that every element not in $P$ is prime to $B$. If $R$ satisfies the ascending chain condition on ideals, then $u(A, P)=A::(x)$ for some $x \notin P$. In other words, $u(A, P)$ is the unique maximal element in the set $\{A:: X \mid X \nsubseteq P\}$.

2. The example. Let $p$ be a positive prime integer and let $G$ be the direct sum of the groups $Z_{p}$ and $Z_{p^{2}}$ of integers modulo $p$ and $p^{2}$ respectively. The elements in $G$ will be designated by $(x, y)$ with $x$ in $Z_{p}$ and $y$ in $Z_{p^{2}}$. In the full ring of endomorphisms of $G$ consider the subring $R$ generated by the four endomorphisms $\alpha, \beta, \gamma$, and $\delta$ defined as follows:

$$
\begin{array}{ll}
\alpha(x, y)=(x, 0), & \beta(x, y)=(y, 0), \\
\gamma(x, y)=(0, p x), & \delta(x, y)=(0, y) .
\end{array}
$$

Elements of $R$ are linear combinations of these four endomorphisms with integral coefficients subject to the restrictions $p \alpha=p \beta$ $=p \gamma=p^{2} \delta=0$. Multiplication is determined by distributive laws and the multiplication table in Diagram 1.

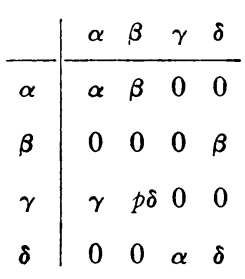

Diagram 1

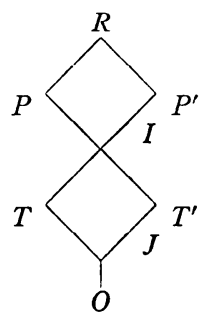

Diagram 2

\begin{tabular}{l|llllllll} 
& $O$ & $J$ & $T$ & $T^{\prime}$ & $I$ & $P$ & $P^{\prime}$ & $R$ \\
\hline$O$ & $R$ & $P$ & $P$ & $T^{\prime}$ & $T^{\prime}$ & $T^{\prime}$ & $O$ & $O$ \\
$J$ & $R$ & $R$ & $P$ & $P^{\prime}$ & $I$ & $T^{\prime}$ & $T$ & $J$ \\
$T$ & $R$ & $R$ & $R$ & $P^{\prime}$ & $P^{\prime}$ & $P^{\prime}$ & $T$ & $T$ \\
$T^{\prime}$ & $R$ & $R$ & $P$ & $R$ & $P$ & $T^{\prime}$ & $P$ & $T^{\prime}$ \\
$I$ & $R$ & $R$ & $R$ & $R$ & $R$ & $P^{\prime}$ & $P$ & $I$ \\
$P$ & $R$ & $R$ & $R$ & $R$ & $R$ & $R$ & $P$ & $P$ \\
$P^{\prime}$ & $R$ & $R$ & $R$ & $R$ & $R$ & $P^{\prime}$ & $R$ & $P^{\prime}$ \\
$R$ & $R$ & $R$ & $R$ & $R$ & $R$ & $R$ & $R$ & $R$
\end{tabular}

Diagram 3 
An ideal $A$ of $R$ containing the element $a \alpha+b \beta+c \gamma+d \delta$ must also contain $a \alpha+b \beta, d \beta, a \gamma+b p \delta, c \gamma+d \delta, a \alpha+c \gamma, a \beta+c p \delta, d \gamma$, and $b \beta+d \delta$. By considering all possible choices of $a, b, c$, and $d$, we obtain principal ideals generated by $a \alpha+b \beta+c \gamma+d \delta$; and this exhausts all ideals of $R$. If we denote the linear span of $\sigma, \rho, \cdots$ by $[\sigma, \rho, \cdots]$, then what follows is a list of all ideals of $R$.

$$
\begin{aligned}
& R, \quad P=[\alpha, \beta, \gamma, p \delta], \quad P^{\prime}=[\beta, \gamma, \delta], \quad I=[\beta, \gamma, p \delta], \\
& T=[\beta, p \delta], \quad T^{\prime}=[\gamma, p \delta], \quad J=[p \delta],
\end{aligned}
$$

Diagrams 2 and 3 show the ideal lattice of $R$ and the right residuation table for ideals of $R$.

2.1. Primary decomposition theory for $R$. On close inspection of the right residuation table in Diagram 3, we find that the only prime ideals of $R$ are $R$ itself and the two maximal ideals $P$ and $P^{\prime}$. Obtain then the radical of each ideal as the intersection of minimal prime divisors; and check from the table that the only primary ideals are those prime ideals. Accordingly the ideals $I, P, P^{\prime}$, and $R$ possess primary decompositions, namely $P \cap P^{\prime}, P, P^{\prime}$ and $R$ respectively; while the ideals $O, J, T, T^{\prime}$ are indecomposable.

2.2. Tertiary decomposition theory for $R$. The ideals $O, T^{\prime}, T$, $P^{\prime}, P$, and $R$ are $\cap$-irreducible and therefore are tertiary. Although $I$ and $J$ are not tertiary, they have the tertiary decompositions $P \cap P^{\prime}$ and $T \cap T^{\prime}$ respectively. The tertiary radicals and associated primes of respective ideals are shown in Diagram 4.

2.3. Upper components. The upper $P$-component $u(A, P)$ of an ideal $A$, as pointed out in $\S 1$, is the unique maximal element of the form $A:: X$ where $X \Phi P$, and this can be obtained directly from the right residuation table. Similarly we can find the upper components of ideals corresponding to $P^{\prime}$ and $R$ and these are listed in the following table. It should be mentioned that the presence of an asterisk in the last three rows indicates that the prime ideal is not prime to the corresponding upper component.

\begin{tabular}{l|cccccccc}
\hline & $O$ & $J$ & $T$ & $T^{\prime}$ & $I$ & $P$ & $P^{\prime}$ & $R$ \\
\hline Radical & $I$ & $I$ & $I$ & $I$ & $I$ & $P$ & $P^{\prime}$ & $R$ \\
Primary decomposition & $\frac{P}{P}$ & $\frac{I}{2}$ & $\frac{P}{P^{\prime}}$ & $\frac{T}{P^{\prime}}$ & $P P^{\prime}$ & $P$ & $P^{\prime}$ & $R$ \\
Tertiary radical & $P$ & $T \cap T^{\prime}$ & $T$ & $T^{\prime}$ & $P \cap P^{\prime}$ & $P$ & $P^{\prime}$ & $R$ \\
Tertiary decomposition & $O$ & $P$ & $P^{\prime}$ & $R$ \\
Associated primes & $P$ & $P, P^{\prime}$ & $P$ & $P^{\prime}$ & $P, P^{\prime}$ & $P$ & $P^{\prime}$ & $R$ \\
Upper $P$-component & $O^{*}$ & $T^{*}$ & $T^{*}$ & $P^{*}$ & $P^{*}$ & $P^{*}$ & $R$ & $R$ \\
Upper $P^{\prime}$-component & $T^{\prime *}$ & $T^{*}$ & $P^{\prime *}$ & $T^{\prime *}$ & $P^{\prime *}$ & $R$ & $P^{\prime *}$ & $R$ \\
Upper $R$-component & $O$ & $J$ & $T$ & $T^{\prime}$ & $I$ & $P$ & $P^{\prime}$ & $R$ \\
\hline
\end{tabular}


2.4. Conclusion. The ideals $P$ and $P^{\prime}$ are associated primes of $J$ and are the only prime ideals not prime to the respective upper components of $J$; yet $J$ possesses no primary decompositions. Murdoch's conjecture is not applicable to the ideal $J$. As we shall see in the next section, this happens solely due to the fact that there are ideals (namely $O, T$, and $T^{\prime}$ ) for which not all the prime ideals not prime to the respective upper components are associated primes.

3. Rings with Noetherian ideal theory. Following Curtis [3, p. 695] we shall call a ring $R$ a ring having a Notherian ideal theory if $R$ satisfies the ascending chain condition on ideals and every ideal of $R$ is an intersection of a finite number of primary ideals. ${ }^{2}$ Such an intersection is called a primary decomposition. It can be verified easily that every primary decomposition can be reduced to a normal one, i.e., an irredundant intersection whose primary components have distinct radicals.

Riley, in response to the question as to when a ring may have a Noetherian ideal theory, has given the following criterion: A right Noetherian ring $R$ with an identity has a Noetherian ideal theory if, and only if it has the Artin-Rees property, namely, given any two ideals $A$ and $B$ of $R, A B \supseteq A^{n} \cap B$ for sufficiently large $n$ [8, p. 195]. This property has also been adopted by Ward and Dilworth [9, Theorem 11.1] to establish the existence of Noether lattices.

3.1. Lemma. If $R$ has the Artin-Rees property, then $t$-rad $A \subseteq \operatorname{rad} A$ for every ideal $A$ of $R$. The converse is true if $R$ satisfies the ascending chain condition on ideals.

Proof. Let $X$ be an ideal of $R$ such that $Y=A$ whenever $Y \cap(A:: X)$ $=A$. By the Artin-Rees property, $X^{n} \bigcap(A:: X) \subseteq X(A:: X) \subseteq A$ for sufficiently large $n$. Since the lattice $L(R)$ is modular,

$$
\left(A+X^{n}\right) \cap(A:: X)=A+\left[X^{n} \cap(A:: X)\right]=A .
$$

Thus $A+X^{n}=A$ or $X^{n} \subseteq A$. This shows that $t$-rad $A \subseteq \operatorname{rad} A$. To prove the converse, we observe that under the hypotheses every ideal of $R$ contains a power of its tertiary radical. If $A B=T_{1} \cap T_{2} \cap \cdots \cap T_{k}$ is a tertiary decomposition of $A B$, then for each $i$ either $B \subseteq T_{i}$ or $A \subseteq t$-rad $T_{i}$. In the latter case, $T_{i}$ contains a power of $A$. We may

2 Except for the difference in left-right terminology, Murdoch's definition of primary ideal is equivalent to that of Curtis. This has been proved by Curtis for the case where $R$ satisfies the ascending chain condition on right ideals. The critical step in his proof is an application of Levitzki's theorem in showing that every ideal contains a power of its radical. This can also be accomplished by $[6$, Theorem 10$]$. 
choose $n$ large enough that $A^{n} \cap B \subseteq T_{i}$ for every $i$ and hence $A^{n} \cap B \subseteq A B$.

The Artin-Rees property has an intimate relation to an interesting identity on ideal in a commutative Noether ring obtained by Barnes and Cunnea, namely, given any two ideals $A$ and $B$ of $R$,

$$
A=\left(A+B^{n}\right) \cap\left(A:: B^{n}\right)
$$

for sufficiently large $n[2$, Lemma 1]. [We shall refer to this as BarnesCunnea property.] In fact

3.2. Lemma. The Barnes-Cunnea property and the Artin-Rees property are equivalent. Both properties imply the following ascending chain condition on right quotients: For any two ideals $A$ and $B$ there exists a positive integer $k$ such that $A:: B^{k}=A:: B^{k+1}=\cdots$.

Proof. Let $R$ be a ring having the Barnes-Cunnea property and let $A$ and $B$ be two ideals of $R$. Then for sufficiently large $n, A B$ $=\left(A B+A^{n}\right) \cap\left(A B:: A^{n}\right) \supseteq A^{n} \cap B$. Conversely if $R$ has the ArtinRees property, then there exists some $k$ such that $A \supseteq B(A:: B)$ $\supseteq B^{k} \cap(A:: B)$. For any $m \geqq k, A:: B^{m} \supseteq\left[B^{k} \cap(A:: B)\right]:: B^{m}$ $=\left(B^{k}:: B^{m}\right) \cap\left[(A:: B):: B^{m}\right]=R \cap\left(A:: B^{m+1}\right)=A:: B^{m+1}$. Thus $A:: B^{k}=A:: B^{k+1}=\cdots$ By Artin-Rees property again, $A \supseteq B^{k}\left(A:: B^{k}\right) \supseteq B^{n} \cap\left(A:: B^{k}\right)=B^{n} \cap\left(A:: B^{n}\right)$ where $n \geqq k$. In virtue of modularity of the lattice $L(R), A=A+\left[B^{n} \cap\left(A:: B^{n}\right)\right]$ $=\left(A+B^{n}\right) \cap\left(A:: B^{n}\right)$.

3.3. THEOREM. Each of the following conditions is necessary and sufficient for a ring $R$ with ascending chain condition on ideals to have a Noetherian ideal theory:

(1) For any ideal $A$ of $R$, every prime ideal $P$ which is not prime to the upper $P$-component $u(A, P)$ occurs as an associated prime of $A$.

(2) For any ideal $A$ of $R, t-\operatorname{rad} A \subseteq \operatorname{rad} A$.

(3) $R$ has the Artin-Rees property.

(4) $R$ has the Barnes-Cunnea property.

Proof. If $R$ has a Noetherian ideal theory, then (1) follows from [6, Theorem 18] and the fact that every primary ideal is tertiary. In view of the proof of [7, Theorem 17], (1) implies (2). Suppose now $R$ has the Barnes-Cunnea property. To show that $R$ has a Noetherian ideal theory it suffices to prove that every $\cap$-irreducible ideal is primary. Let $A$ be a nonprimary ideal of $R$. Then there exist ideals $B$ and $C$ of $R$ such that $B C \subseteq A$ but neither $C$ nor any power of $B$ is contained in $A$. By (4), $A=\left(A+B^{n}\right) \cap\left(A:: B^{n}\right)$ for some $n$. 
This shows that $A$ is properly reducible and therefore completes the proof.

Incidentally, Murdoch's condition involves one of the many characterizations of associated primes of ideals. Aside from the existing characterizations given by Murdoch [6, Theorem 18], Curtis [3, Definition 1.10 and Theorem 1.17(a)], Lesieur and Croisot [5, Theorem 8.4], we shall describe a method of finding associated primes, whose commutative counterpart has already been discovered by Barnes and Cunnea [2, pp. 182-3].

Assume henceforth that $R$ is a ring with a Noetherian ideal theory. If $Q$ is a $P$-primary divisor of an ideal $A$ of $R$, then for sufficiently large $e, A \subseteq A+P^{e} \subseteq u\left(A+P^{e}, P\right) \subseteq Q$ where $A+P^{e}$ is quasi $P$-primary and $u\left(A+P^{e}, P\right)$ is $P$-primary. The following theorem can then be derived easily.

3.4. THEOREM. Let $R$ be a ring with a Notherian ideal theory and let $P_{1}, P_{2}, \cdots, P_{n}$ be associated primes of an ideal $A$ of $R$ such that $P_{1}, \cdots, P_{k}(k \leqq n)$ are the minimal prime divisors of $A$. Then for sufficiently large $e$,

$$
A=\left(A+P_{1}^{e}\right) \cap\left(A+P_{2}^{\epsilon}\right) \cap \cdots \cap\left(A+P_{k}^{e}\right)
$$

is an irredundant quasi primary decomposition of $A$; and

$$
A=u\left(A+P_{1}^{e}, P_{1}\right) \cap u\left(A+P_{2}^{e}, P_{2}\right) \cap \cdots \cap u\left(A+P_{n}^{e}, P_{n}\right)
$$

is a normal primary decomposition of $A$.

Now let $P$ be a minimal prime divisor of $A$. Then by $\left(^{*}\right), u(A, P)$ contains a power of $P$ and is therefore $P$-primary. If $Q$ is any primary divisor of $A$ contained in $u(A, P)$, then $P=\operatorname{rad} Q$ and so $u(A, P) \subseteq Q$. Hence $u(A, P)$ is a minimal primary divisor of $A$. Conversely, if $u(A, P)$ is $P$-primary, then for any minimal prime divisor $P^{\prime}$ of $A$ contained in $P, u(A, P)=u\left(A, P^{\prime}\right)$. Hence $P=P^{\prime}$, a minimal prime divisor of $A$. This proves a part of the following lemma, the rest is obvious.

3.5. Lemma. Let $R$ be a ring with a Noetherian ideal theory and let $P$ be a prime divisor of an ideal $A$. Then the following statements are equivalent:

(1) $P$ is a minimal prime divisor of $A$.

(2) $u(A, P)$ is $P$-primary.

(3) $u(A, P)=u\left(A+P^{e}, P\right)$ for sufficiently large e. Each of the statements (1)-(3) implies

(4) $u(A, P)$ is a minimal primary divisor of $A$. 
In view of the above lemma,

$$
A_{1}=\cap\{u(A, P) \mid P \text { is a minimal prime divisor of } A\}
$$

is a primary decomposition. Define $A_{k}(k \geqq 2)$ inductively as follows: $A_{k}=\bigcap\left\{u(A, P) \mid P\right.$ is a minimal prime divisor of $\left.A:\left(A_{1} \cap \cdots \cap A_{k-1}\right)\right\}$. Let $A=Q_{1} \cap Q_{2} \cap \cdots \cap Q_{n}$ be a normal primary decomposition of $A$ where $\operatorname{rad} Q_{i}=P_{i}$ and $P_{1}, \cdots, P_{q}$ are the minimal prime divisors of $A$. Then $Q_{i}=u\left(A, P_{i}\right)$ for $i=1,2, \cdots, q$, and since none of the $Q_{j}(j>q)$ contains $A_{1}$, we have

$$
A: A_{1}=\left(Q_{q+1}: A_{1}\right) \cap \cdots \cap\left(Q_{n}: A_{1}\right)
$$

which is a primary decomposition of $A: A_{1}$. Note that $\operatorname{rad}\left(Q_{j}: A_{1}\right)$ $=\operatorname{rad} Q_{j}=P_{j}$. The minimal prime divisors of $A: A_{1}$ must occur among the $P_{j}(j>q)$. Assume without loss of generality that these are $P_{q+1}, \cdots, P_{r}$. For each $q<j \leqq r$,

$$
u\left(A, P_{j}\right)=Q_{j} \cap\left[\cap\left\{Q_{i} \mid 1 \leqq i \leqq q \text { and } P_{i} \subseteq P_{j}\right\}\right] .
$$

Hence $A_{1} \cap A_{2}=Q_{1} \cap Q_{2} \cap \cdots \cap Q_{r}$. If $r<n$, consider then the primary decomposition

$$
A:\left(A_{1} \cap A_{2}\right)=\left(Q_{r+1}:\left(A_{1} \cap A_{2}\right)\right) \cap \cdots \cap\left(Q_{n}:\left(A_{1} \cap A_{2}\right)\right) .
$$

It can be proved that $A_{1} \cap A_{2} \cap A_{3}=Q_{1} \cap \cdots \cap Q_{s}$ for some $s>r$. Continue in this way we arrive, after a finite number of steps, at

$$
A:\left(A_{1} \cap A_{2} \cap \cdots \cap A_{m}\right)=R .
$$

So, in case $R$ has an identity, $A=A_{1} \cap \cdots \cap A_{m}=Q_{1} \cap \cdots \cap Q_{t}$ for some $t \leqq n$. Since the original primary decomposition of $A$ is irredundant, $t=n$.

If $P$ is an associated prime of $A$ and if $P_{1}, P_{2}, \cdots, P_{h}$ are associated primes of $A$ such that $A \subseteq P_{1} \subset P_{2} \subset \cdots \subset P_{h}=P$, then we call $\left(P_{1}, P_{2}, \cdots, P_{h}\right)$ a chain of $P$ over $A$ of length $h$. The unique maximal length of all the chains of $P$ over $A$ is called the rank of $P$ over $A$.

3.6. Theorem. Let $R$ be a ring with an identity and a Noetherian ideal theory and let $A$ be an ideal of $R$. Define $A_{0}=R$ and $A_{k}=\cap\{u(A, P) \mid P$ is a minimal prime divisor of $\left.A:\left(A_{0} \cap \cdots \cap A_{k-1}\right)\right\}(k=1,2, \cdots)$. Then $A_{1} \supset A_{2} \supset A_{3} \supset \cdots \supset A_{m}=A$ for some $m$ and the associated primes and of $A$ of rank $k$ are precisely the minimal prime divisors of $A: A_{k-1}(k=1,2, \cdots, m)$.

\section{REFERENCES}

1. W. E. Barnes, Primal ideals and isolated components in noncommutative rings, Trans. Amer. Math. Soc. 82 (1956), 1-16. 
2. W. E. Barnes and W. M. Cunnea, Ideal decompositions in Noetherian rings, Canad. J. Math. 17 (1965), 178-184.

3. C. W. Curtis, On additive ideal theory in general rings, Amer. J. Math. 74 (1952), $687-700$.

4. L. Lesieur et R. Croisot, Théorie Noetherrienne des anneaux, des demi-groupes et des modules dans le cas non commutatif. II, Math. Ann. 134 (1958), 458-476.

5. - Algèbre Noethérienne non commutative, Gauthier-Villars, Paris, 1963.

6. D. C. Murdoch, Contributions to noncommutative ideal theory, Canad. J. Math. 4 (1952), 43-57.

7. - Subrings of the maximal ring of quotients associated with closure operations, Canad. J. Math. 15 (1963), 723-743.

8. J. A. Riley, Axiomatic primary and tertiary decomposition theory, Trans. Amer. Math. Soc. 105 (1962), 177-201.

9. M. Ward and R. P. Dilworth, Residuated lattices, Trans. Amer. Math. Soc. 45 (1939), 335-354.

\section{State University of New York at Buffalo}

